\title{
Large eddy simulation for jet noise: the importance of getting the boundary layer right
}

\author{
Guillaume A. Brès*, \\ Cascade Technologies Inc., Palo Alto, CA 94303 \\ Vincent Jaunet† Maxime Le Rallic $\ddagger$ Peter Jordan§ \\ Institut PPRIME, UPR-CNRS-3346, Université de Poitiers, France \\ Tim Colonius, \\ California Institute of Technology, Pasadena, CA 91125 \\ and \\ Sanjiva K. Lelell \\ Stanford University, Stanford, CA 94305
}

\begin{abstract}
Large eddy simulations of an isothermal Mach 0.9 jet issued from a convergent-straight nozzle are performed at Reynolds number $1 \times 10^{6}$. The flow configuration and operating conditions match the companion experiment conducted at the PPRIME Institute, Poitiers. To replicate the effects of the boundary layer trip present in the experiment and to ensure a turbulent jet, localized adaptive mesh refinement, synthetic turbulence, and wall modeling are used inside the nozzle. This leads to fully turbulent nozzle-exit boundary layers and results in significant improvements for the flow field and sound predictions, compared to those obtained from the typical approach based on laminar flow assumption in the nozzle. The far-field noise spectra now match the experimental measurements to within $0.5 \mathrm{~dB}$ for relevant angles and frequencies. As a next step toward better understanding of turbulent jet noise, the large database collected during the simulation is currently being used for reduced order modeling and wavepacket analysis.
\end{abstract}

\section{Nomenclature}

c $\quad$ Speed of sound

$D \quad$ Nozzle exit diameter

NPR Nozzle pressure ratio

$N T R$ Nozzle temperature ratio

$P \quad$ Pressure

Re Reynolds number

St Strouhal number $f D / U_{j}$

$T$ Temperature

$t \quad$ Time

$x, y, z \quad$ Cartesian coordinates

$\mu \quad$ Dynamic viscosity $\phi \quad$ Jet inlet angle

$\rho \quad$ Density

Subscript

$\infty \quad$ Free-stream property

$t \quad$ Total (stagnation) property

$j \quad$ Fully-expanded jet conditions

Superscript

' Disturbance quantity

- $\quad$ Time average

\footnotetext{
${ }^{*}$ Senior Research Scientist

${ }^{\dagger}$ Postdoctoral Scholar

${ }^{\ddagger}$ Graduate Student

$\S$ Research Scientist

"Professor, Dept. of Mechanical Engineering, AIAA Associate Fellow

" Professor, Dept. of Mechanical Engineering and Dept. of Aeronautics \& Astronautics
} 


\section{Introduction}

To improve understanding and modeling of the turbulent sources of sound in subsonic jets, extensive experimental and numerical databases were generated for a Mach 0.9 turbulent jet. The experiments conducted at PPRIME Institute include characterization of the nozzle-exit boundary layers, flow field PIV, and both near-field and far-field pressure measurements on large microphone arrays. As part of the Center for Turbulence Research (CTR) Summer Program 2014 at Stanford University, a companion large eddy simulation (LES) was performed using the compressible flow solver "Charles" developed at Cascade Technologies. ${ }^{1}$ One of the challenging aspects of the present configuration is that transition to turbulence is forced in the experiment using a boundary layer trip inside the nozzle. From past experimental ${ }^{2-4}$ and numerical studies, ${ }^{5,6}$ the state of the nozzle-exit boundary layer is known to be a key parameter for the flow development and noise characteristics of a jet. However, because of the computational cost of simulating high Reynolds number wall-driven turbulence, the nozzle boundary layer is typically assumed to be laminar or weakly disturbed in most jet simulations. This approach often leads to enhanced laminar-to-turbulent shear-layer transition and increased noise due to vortex pairing.

The present simulations leveraged recent research efforts focusing on this important aspect of the jet noise problem: the modeling of the nozzle interior flow and its effects on the nozzle-exit boundary layer, the jet plume, and ultimately the acoustic field. ${ }^{7,8}$ Here, localized adaptive mesh refinement, synthetic turbulence, and wall modeling are used inside the nozzle to ensure fully turbulent profiles at the nozzle exit. These modeling approaches, as well as the experimental configuration and the numerical setup, are briefly reviewed in Section II. Then, in Section III, results from a series of preliminary large eddy simulations are discussed to highlight the improvements associated with the different modeling applied inside the nozzle. Details of the main LES database generated are presented in Section IV, including complete comparisons with the available experimental measurements.

\section{Flow configuration and numerical methods}

\section{A. Experimental setup}

The study focuses on an isothermal Mach 0.9 jet issued from contoured convergent-straight nozzle of exit diameter $D=50 \mathrm{~mm}$. The resulting diameter-based Reynolds number is approximately $10^{6}$. Transition is forced using an azimuthally homogeneous carborundum strip located $128 \mathrm{~mm}$ upstream of the nozzle exit plane. The experiments were performed at the "Bruit et Vent" jet-noise facility of the PPRIME Institute at the Centre d'Études Aérodynamiques et Thermiques (CEAT), Poitiers, France (see Figure 1). The experimental diagnostics included hot-wire and laser doppler velocimetry (LDV) systems for measurement of the exit velocity profiles, particle image velocimetry (PIV) for more extensive measurement of the jet plume, a 48-microphone near-field cage array for the eduction of wavepacket signatures, and an axially traversable, 18-microphone, azimuthal array providing measurements of the sound field on a cylindrical surface of radius $r=14.3 D$. Pressure measurements are also performed in the far field at a constant distance of $50 D$ from the nozzle exit using a single microphone every $10^{\circ}$, from inlet angle $90^{\circ}$ (i.e., normal to nozzle-exit plane) to $160^{\circ}$ (i.e., downstream of nozzle exit). Details on the post-processing of the noise data are presented in Appendix.

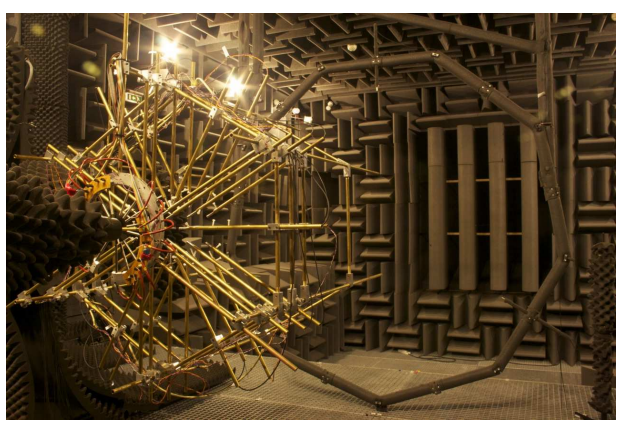

(a) Near-field cage array

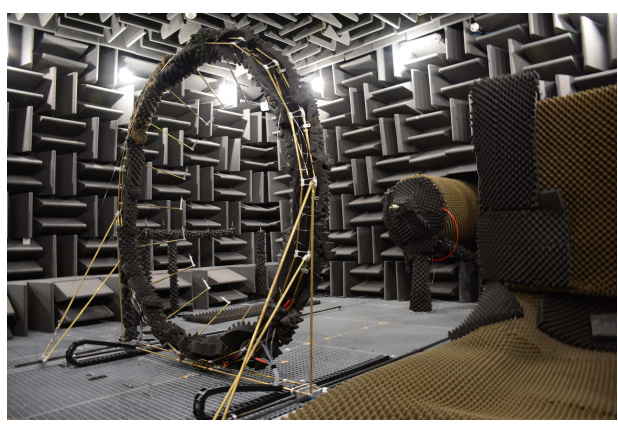

(b) Azimuthal array

Figure 1. Experimental set-up in anechoïc jet-noise facility 
The PIV system consisted of 2 Photron SAZ cameras and a 532nm 6mJ Continuum MESA PIV laser, placed on a traverse parallel to the jet axis. Both the jet flow and the surrounding air were seeded using glycerin smoke particles, whose diameter lay in the range $1-2 \mu m$, thus sufficiently small to follow the velocity fluctuations of interest in this paper. The image acquisition was performed at $20 \mathrm{kHz}$ (10000 PIV samples a second) at a resolution of $1024 \times 1024$ pixels. The time between the two laser pulses was set according to the local velocity amplitude and to the laser sheet width (which was set at $2 \mathrm{~mm}$ ), and ranged between 4 and $5 \mu \mathrm{s}$. For each acquisition, 42,000 image pairs were acquired. PIV calculations were carried out using a commercial software, and a multipass iterative PIV algorithm. ${ }^{9}$ The PIV interrogation area size was set to $32 \times 32$ pixels for the first pass, decreased at $16 \times 16$ pixels with an overlap of $50 \%$ between two neighboring interrogation areas. Five different acquisition runs allowed to scan the jet in between $x / D=0$ and $x / D=20$. For each run, the field of view was adjusted either by moving the camera away from the jet or by changing the lens, in order to account for the development of the flow.

\section{B. Numerical setup}

In this work, the jet is investigated with the high-fidelity LES framework developed at Cascade Technologies. The framework is composed of the pre-processing mesh adaptation tool "Adapt", the compressible flow solver "Charles," and post-processing tools for far-field noise predictions based on an efficient massively-parallel implementation ${ }^{10}$ of the frequency-domain permeable formulation ${ }^{11}$ of the Ffowcs Williams \& Hawkings ${ }^{12}$ (FW-H) equation.

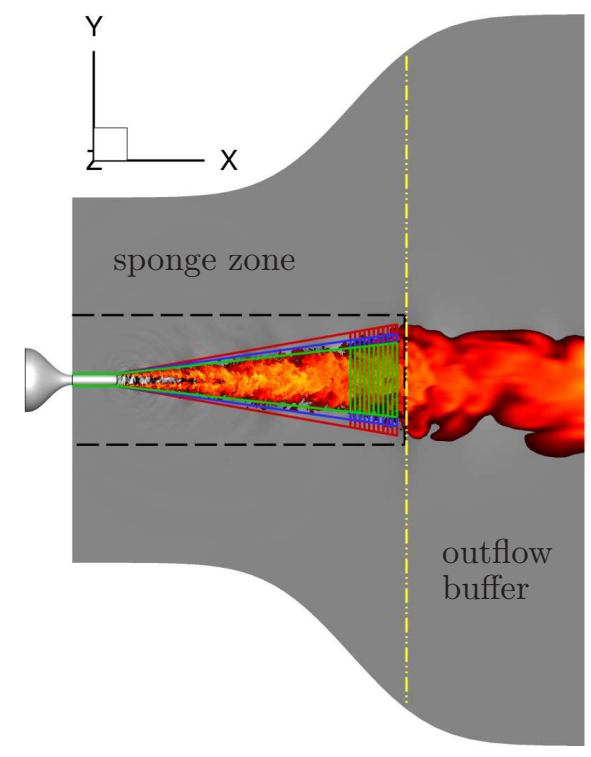

Figure 2. Schematic of the flow configuration and numerical setup

The numerical setup, the FW-H surfaces used to compute the far-field noise, and the baseline methodologies are similar to previous jet studies with the flow solver Charles. ${ }^{7,8,10,13-15}$ The nozzle pressure ratio and nozzle temperature ratio are $N P R=P_{t} / P_{\infty}=1.7$ and $N T R=T_{t} / T_{\infty}=1.15$, respectively, and match the experimental conditions. Here, the subscript $t$ and $\infty$ refer to the stagnation (total) property and freestream (ambient) quantity, respectively. The jet is isothermal $\left(T_{j} / T_{\infty}=1.0\right)$, and the jet Mach number is $M_{j}=U_{j} / c_{j}=0.9$, where $U_{j}$ is the mean (time-averaged) streamwise jet velocity and the subscript $j$ refer to the jet properties. For both experiment and simulation, the Reynolds number is $R e=\rho_{j} U_{j} D / \mu_{j} \approx 1 \times 10^{6}$.

The round nozzle geometry (with exit centered at $(0,0,0)$ ) is explicitly included in the axisymmetric computational domain, which extends from approximately $-10 D$ to $50 D$ in the streamwise (x) direction and flares in the radial direction from $20 D$ to $40 D$ (Figure 2). Note that a very slow coflow at Mach number $M_{\infty}=0.009$ is imposed outside the nozzle in the simulation $\left(M_{\infty}=0\right.$ in the experiment), to prevent any spurious recirculation and facilitate flow entrainment. Sponge layers and damping functions are applied to avoid spurious reflections at the boundary of the computational domain. ${ }^{16,17}$ The Vreman ${ }^{18}$ sub-grid model is used to account for the physical effects of the unresolved turbulence on the resolved flow. An extension to the digital filtering technique ${ }^{19}$ for the generation of synthetic turbulence on unstructured grids is currently 


\begin{tabular}{l|c|c|c|c|c|c|c}
\hline Case name & $\begin{array}{r}\text { Mesh size } \\
\left(10^{6} \text { cells }\right)\end{array}$ & $\begin{array}{c}\text { BL } \\
\text { refine- } \\
\text { ment }\end{array}$ & $\begin{array}{c}\text { Synthetic } \\
\text { turbulence } \\
u_{\text {trip }}^{\prime} / u_{\tau}\end{array}$ & $\begin{array}{c}\text { Wall } \\
\text { model }\end{array}$ & $d t c_{\infty} / D$ & $t_{s i m} c_{\infty} / D$ & $\begin{array}{c}\text { Database } \\
\text { sampling } \\
\Delta t c_{\infty} / D\end{array}$ \\
\hline \hline \multicolumn{7}{|c}{ Baseline LES cases } \\
\hline $64 M$ & 10.8 & & & & 0.001 & 300 & \\
\hline \hline & 64.2 & & & & 0.0005 & 300 & \\
\hline LES cases with nozzle interior flow modeling \\
BL16M_Turb2 & 15.9 & $\times$ & & & 0.001 & 300 & \\
BL16M_Turb & 15.9 & $\times$ & 2 & & 0.001 & 300 & \\
BL16M_WM & 15.9 & $\times$ & 0.8 & & 0.001 & 300 & \\
BL16M_WM_Turb2 & 15.9 & $\times$ & & $\times$ & 0.001 & 300 & \\
BL16M_WM_Turb & 15.9 & $\times$ & 2 & $\times$ & 0.001 & 300 & \\
BL69M_WM_Turb & $\mathbf{1 5 . 9}$ & $\times$ & $\mathbf{0 . 8}$ & $\times$ & $\mathbf{0 . 0 0 1}$ & $\mathbf{2 0 0 0}$ & $\mathbf{0 . 2}$ \\
\hline
\end{tabular}

Table 1. Simulation parameters of the different LES performed (bold font: database used during the CTR Summer Program 2014). For all cases, the sampling period of the data recording on the FW-H surface is $\Delta t_{F W H} c_{\infty} / D=0.05$.

being developed by Cascade Technologies in collaboration with Stanford University. When active, synthetic turbulence boundary conditions are used to model the boundary layer trip present in the experiment at $-2.8<x / D<-2.5$ on the internal nozzle surfaces. Based on an estimate of the wall friction velocity $u_{\tau}$ from the baseline LES calculation at the trip location, synthetic turbulence fluctuations were introduced with a prescribed amplitude, $u_{t r i p}^{\prime} / u_{\tau}$. In the baseline simulations, all the solid surfaces are treated as noslip adiabatic wall. When active, the wall model, based on the work of Kawai \& Larsson $^{20}$ and Bodart \& Larsson $^{21,22}$ is applied inside the nozzle, in the straight pipe section between the boundary layer trip and the nozzle exit.

The FW-H surfaces used to compute the far-field noise are presented in figure 2, along with visualization of the instantaneous temperature and pressure field. All three conical FW-H surfaces extends to $x=30 D$, with different spreading rates. For the treatment of the FW-H outflow disk, the method of "end-caps" of Shur et al..$^{23}$ is applied for $x>25 D$, where the complex far-field pressure predicted from eleven $\mathrm{FW}-\mathrm{H}$ surfaces with the same shape but outflow disks at different streamwise locations are phase-averaged.

Table 1 lists the settings and parameters for each LES run considered, including the total simulation time (after the initial transient was removed) $t_{\text {sim }} c_{\infty} / D$, and the data sampling period $\Delta t c_{\infty} / D$ for the cases where the LES database was collected. The main case used during the CTR Summer Program 2014 is highlighted in bold font. The simulations with nozzle interior modeling focused on adaptive isotropic mesh refinement of the internal boundary layer (prefix $B L$ ), synthetic turbulence (prefix Turb), and wall-stress modeling inside the nozzle (prefix $W M$ ) .

\section{Mesh adaptation and refinement}

The same grid adaptation approach used for previous jet studies by Brès et al. ${ }^{7,8,13}$ is applied to the present configuration. The starting point is a coarse "skeletal" grid with a paved core, containing about 0.4 million control volumes. Several embedded zones of refinement are then defined by the user and enforced by the "Adapt" tool. The main refinement zones correspond to the bulk of the mesh (containing the jet plume and fully enclosing the FW-H surfaces used for the far-field noise predictions), the jet potential core, and near-nozzle exit.

For the baseline cases, two grids were generated: a standard mesh containing approximately 10 million unstructured control volumes (see Figure 3(a)), and a refined mesh with 64 million cells, by doubling the resolution in the each refinement zones. Note that for these cases, there is no specific near-wall or nozzle interior refinement, and both grids have exactly the same coarse resolution inside the nozzle. 


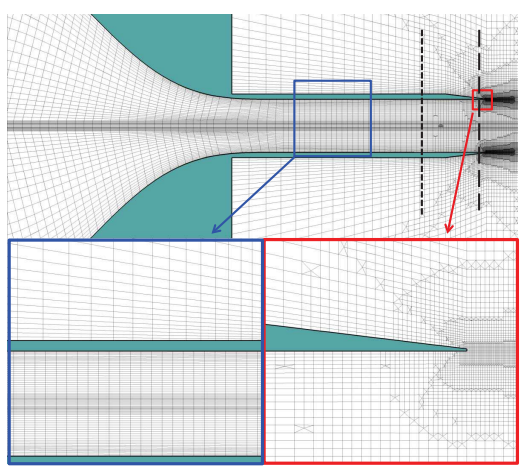

(a) Baseline $10 M$ case

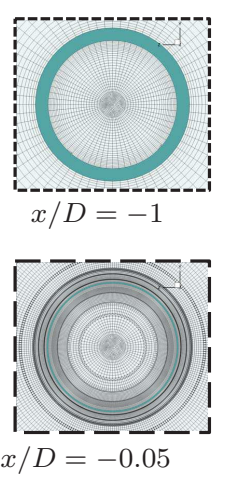

$x / D=-0.05$

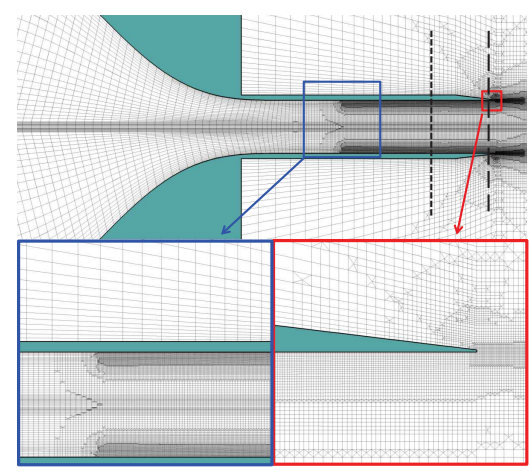

(b) $B L 16 M$ case
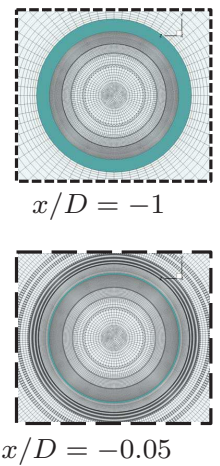

$x / D=-0.05$

Figure 3. Mesh inside the nozzle $(a)$ without and $(b)$ with boundary layer grid isotropic refinement. The blue and red boxes are the zoomed-in views near the boundary layer trip and the nozzle tip, respectively . Cross-sections of the grid inside the nozzle at $x / D=-1$ (short-dash line and box) and near the nozzle exit at $x / D=-0.05$ (long-dash line and box) are also shown.

In contrast, for the simulations involving enhanced modeling of the interior nozzle flow, it can be anticipated that some amount of mesh refinement is also needed inside the nozzle to resolve the large-scale three-dimensional turbulent structures associated with the internal boundary layers. Therefore, isotropic refinement is added to the previous adaptation strategy and applied from the start of the boundary layer trip at $x / D=-2.8$ to the nozzle exit at $x / D=0$, for a constant distance $0.085 D$ from the nozzle wall and with target length scale equal to $0.0075 D$. The distance was chosen based on estimates of the experimental nozzle-exit boundary layer thickness, $\delta_{\exp } / D \approx 0.08$, and the length scale was chosen to yield about $10-20$ LES cells in the boundary layer. The adapted grids with boundary layer refinement now contain approximately 16 million (see Figure 3(b)) and 69 million cells, for the standard and refined cases, respectively. This isotropic refinement strategy effectively results in about 1050 cells in the azimuthal direction near the nozzle internal surface and a finest wall-normal resolution of approximately $0.0035 \mathrm{D}$.

\section{Parametric study on flow modeling inside the nozzle}

In preliminary work prior to the CTR Summer Program 2014, a systematic parametric study of the separate and combined effects of different modeling within the nozzle interior was conducted on the standard mesh, focusing on localized adaptive mesh refinement inside the nozzle, application of synthetic turbulence and wall modeling. At that time, the experimental data available for comparison with LES was limited to the nozzle exit velocity profiles and far-field noise measurements at $50 \mathrm{D}$ from the nozzle exit. The complete analysis will be presented in future publications but the conclusions of the parametric studies and the main results leading to the generation of the large LES database for wavepacket analysis are summarized in the next two sections. To provide consistent comparisons, the same total simulation time $t_{\text {sim }} D / c_{\infty}=300$ was used for the computation of the flow statistics and far-field noise spectra presented in this section.

\section{A. Effects of nozzle interior modeling on flow field results}

Figure 4 shows the comparison of the instantaneous nozzle interior flow with and without modeling. More quantitative comparisons are presented in Figures 5 and 6, for the nozzle-exit profiles and the velocity statistics along the lipline, respectively.

Recall that all the baseline cases have the same operating conditions and essentially the same coarse mesh inside the nozzle. This leads to the same nozzle interior flow field and thin laminar boundary layer inside the nozzle (see top row in figure 4). This qualitative result is confirmed by the inspection of the nozzle exit velocity profiles in figure 5. Both experimental hot-wire measurements and LES results are reported at the same location just downstream of the nozzle exit, at $x / D=0.04$. Note that the very slow coflow $M_{\infty}=0.009$ imposed in the simulation outside the nozzle is the main cause of the slight mismatch in mean velocity for $y / D<-0.5$. For both baseline cases, the time-averaged streamwise velocity profiles are identical and correspond to the typical laminar profile. In contrast, the nozzle-exit boundary layer in the experiment 

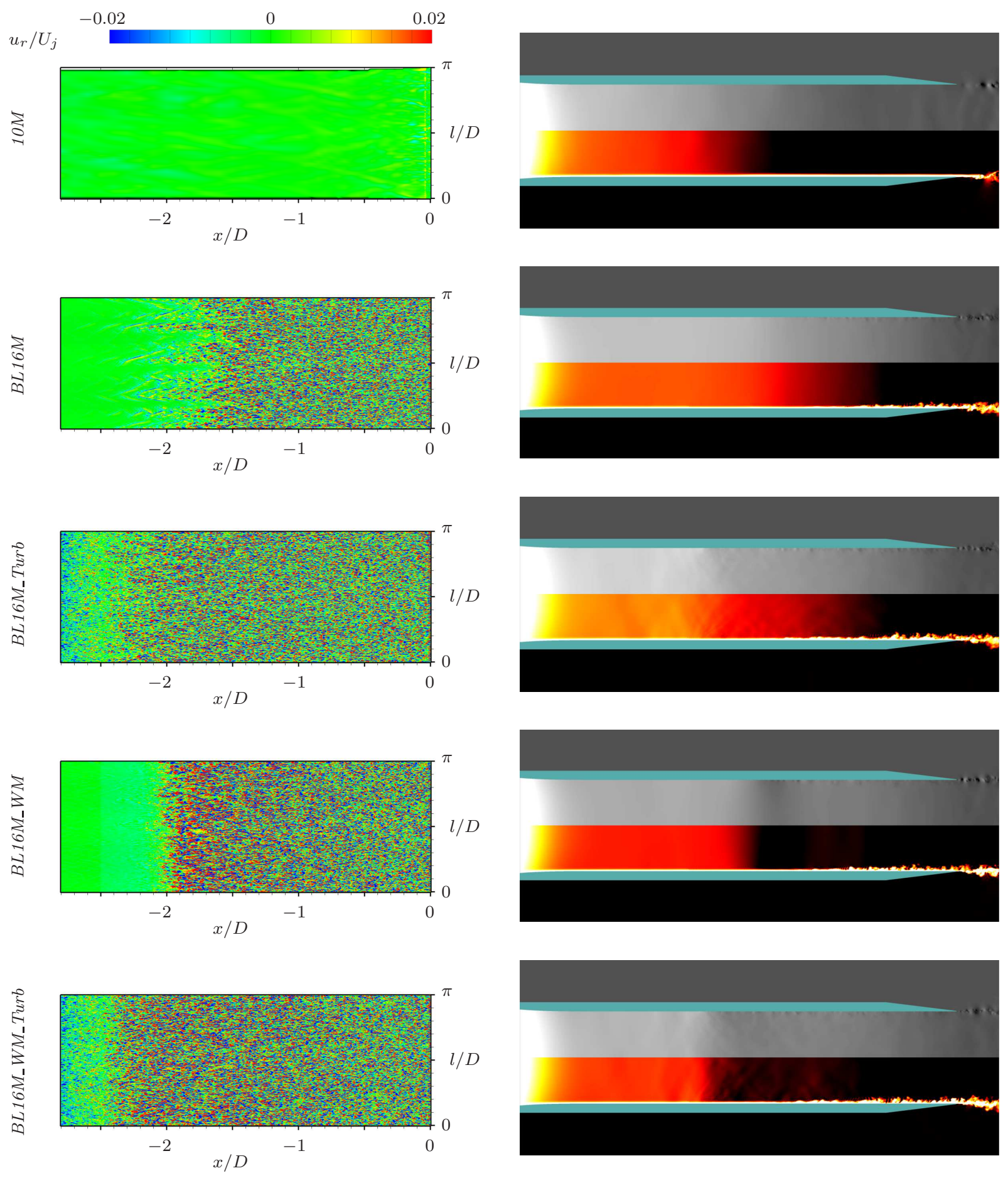

(a) Wall-normal velocity

(b) Pressure and temperature

Figure 4. Zoom-in view of the instantaneous flow field inside the nozzle, for the baseline case $10 M$ (top row) and the LES cases with nozzle interior modeling BL16M (middle row) and BL16M_WM_Turb (bottom row): (a) Wall-normal velocity $u_{r} / U_{j}$ in the first cell near the (unrolled) nozzle interior surface. Recall that the synthetic turbulence is applied for $-2.8 \leq x / D \leq-2.5$, when active; $(b)$ pressure (top half - gray scale) and temperature field (bottom half - red scale) in the mid-section plane $(z=0)$ inside the nozzle . 


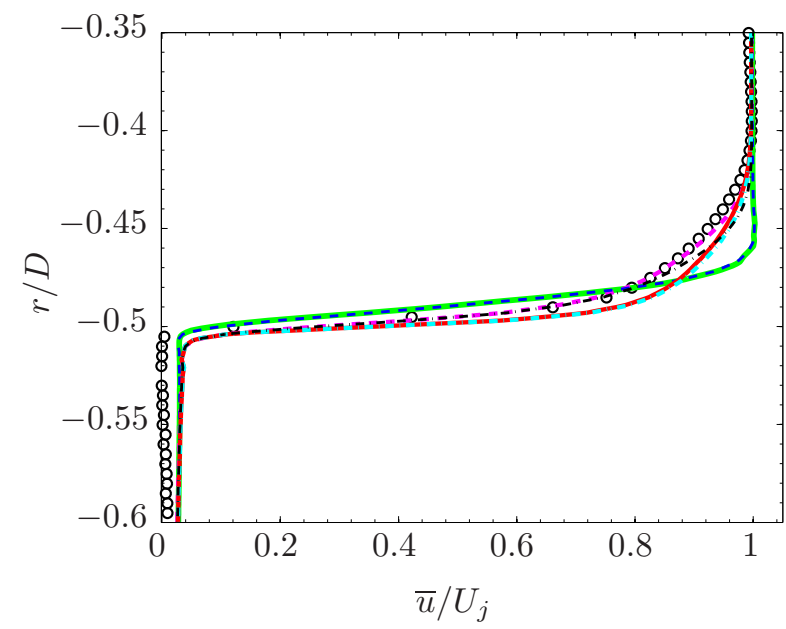

(a) Time-averaged streamwise velocity

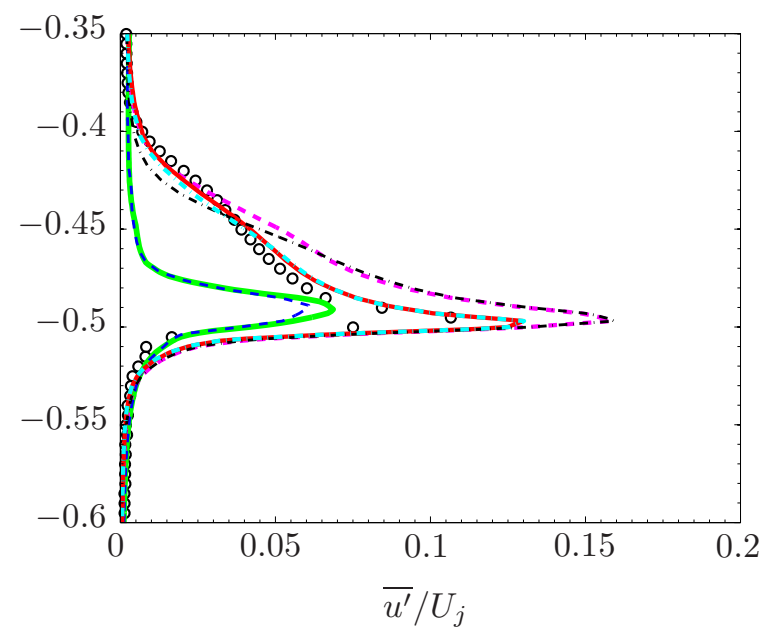

(b) RMS of streamwise velocity

Figure 5. Nozzle-exit boundary layer profiles from experiment ( $\circ$ ), baseline cases $10 M(----)$ and $64 M$ ( ( $)$, and with modeling BL16M ( --—), BL16M-Turb (---), BL16M-WM ( - - ) and BL16M-WM-Turb $(-)$.

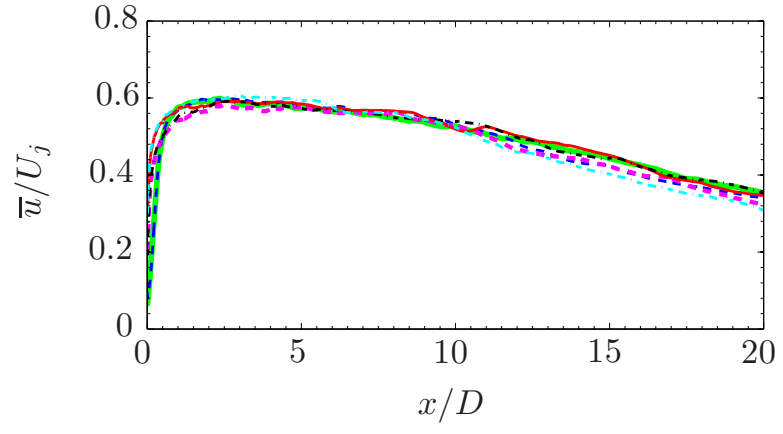

(a) Time-averaged streamwise velocity

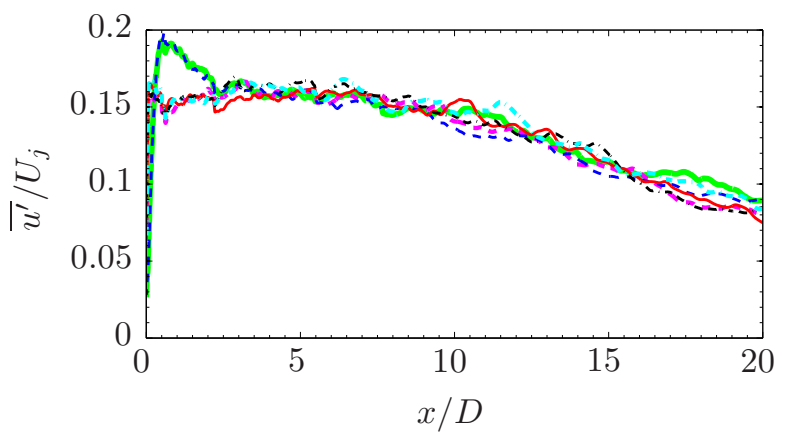

(b) RMS of streamwise velocity

Figure 6. Streamwise velocity statistics along the lipline for the baseline cases $10 M(----)$ and $64 M(-\square)$,

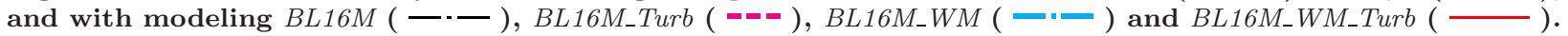

is turbulent, thanks to the azimuthally homogeneous carborundum strip upstream in the pipe. The RMS profiles are therefore largely under predicted for both $10 \mathrm{M}$ and $64 \mathrm{M}$, and there is essentially no velocity fluctuations inside the nozzle for the baseline cases.

In terms of the impact of the nozzle interior modeling, the first conclusion is that all the simulations with isotropic refinement of the boundary layer mesh now display small-scale three-dimensional turbulent structures in the boundary layer inside the nozzle. Depending on the additional modeling applied inside the nozzle (i.e., synthetic turbulence, wall modeling, both, or none), the details of the development of the turbulence inside the nozzle are different. However, the internal flow field within the last $1 \mathrm{D}$ from the nozzle exit look similar in all cases, much like the exit profiles. All the nozzle-exit boundary layers now exhibit turbulent mean and RMS velocity profiles, with much larger fluctuation levels near the wall than in the baseline cases with the thin laminar boundary layer. Overall, the grid adaptation has the most significant impact on the nozzle interior flow field for the present configuration.

Second, the effect of the wall model is also clearly visible and largely beneficial. While the nozzle-exit RMS levels are over-predicted compared to experiment for cases BL16M and BL16M_Turb (see Figure 5(b)), the cases with wall modeling show less of an overshoot and better agreement with the measurements.

Finally, modeling of the experimental trip with synthetic turbulence also slightly improved the interior nozzle flow predictions, but overall had a more limited impact than the two previous items. Two different levels of amplitudes for the synthetic turbulence were tested (see Table 1), and the results show that the nozzle-exit boundary layer profiles and turbulence levels were largely independent of these input parameters. 


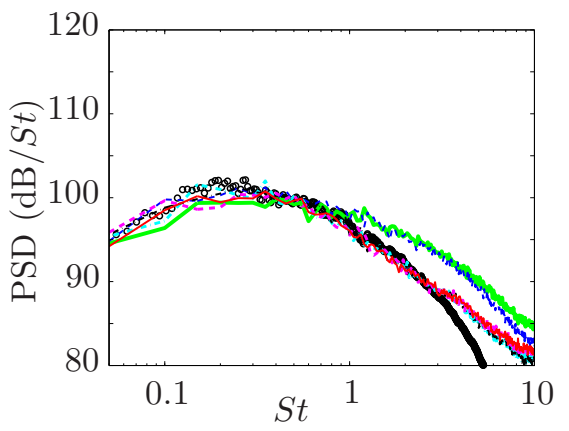

(a) $\phi=90^{\circ}$

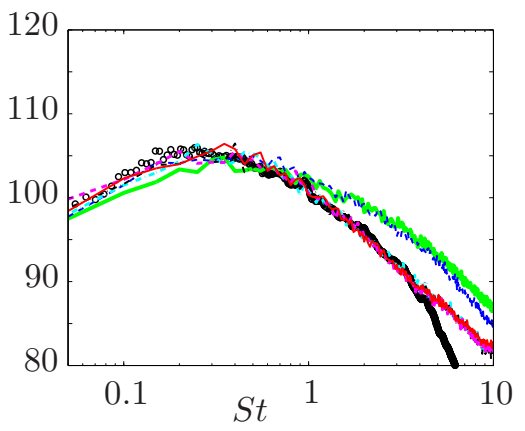

(b) $\phi=120^{\circ}$

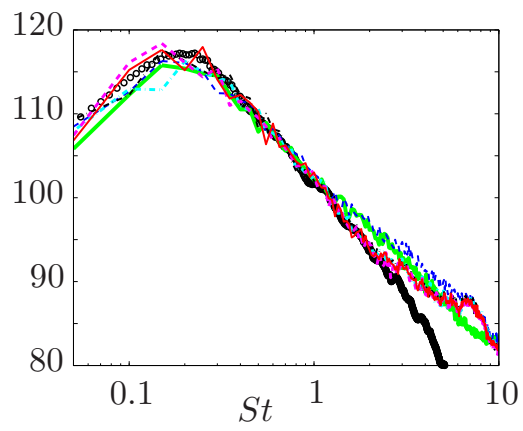

(c) $\phi=150^{\circ}$

Figure 7. Comparison of the noise spectra from experiment ( $\circ)$, baseline LES cases 10M ( ---- ) and $64 M(-)$, and LES cases with modeling BL16M (-- - ), BL16M_Turb (--- ), BL16M-WM( - - ) and $B L 16 M_{-} W M_{-}$Turb ( - ) at $50 D$ from the nozzle exit for selected inlet angles $\phi$.

This trend is similar to the conclusions of Bogey et al. ${ }^{24}$ who reported that their results vary negligibly with the tripping procedure applied inside the nozzle in their simulations.

The velocity statistics along the lipline $(r / D=0.5)$ in Figure 6 also shows improved results for the LES cases with nozzle interior modeling, consistent with previous observations. The most drastic change can be observed in the velocity RMS along the lipline in Figure 6(b) where the fluctuation overshoot around $x=0.5 D$ (related to the shear layer laminar to turbulent transition) is present in both baseline LES, independently of the resolution in the jet plume, and is nearly completely removed with modeling.

\section{B. Effects of nozzle interior modeling on far-field acoustic results}

For all LES cases, calculations of the far-field noise at $50 \mathrm{D}$ from the nozzle exit were performed for the three different FW-H surfaces shown in figure 2: a "tight" surface $S_{0}$ (green outline in figure 2), a "default" surface $S_{1}$ (blue outline) and a "loose" surface $S_{2}$ (red outline). Similarly to previous studies, ${ }^{10}$ the results showed nearly identical spectra over the whole frequency range for the three FW-H surfaces. This lack of sensitivity to the FW-H surface location is a good indication of the robustness of the methods, and only the results from the surface $S_{1}$ are presented in this paper.

Figures 7 and 8 show the comparisons between experiment and LES cases with and without nozzle interior modeling, for the power spectral density (PSD) at the different experimental microphones, and the Overall Sound Pressure Level (OASPL) directivity, respectively. The PSD is reported in $\mathrm{dB} / \mathrm{St}$, versus frequency in Strouhal $S t=f D / U_{j}$, following the same non-dimensionalization than the experiment (see Appendix). Additional averaging is performed on the computational results, to compensate for the relatively short time signal in simulation compared to experiment. First, to leverage the azimuthal symmetry in the jet plume, 36 equally-spaced microphones are distributed along the azimuthal angle, for each inlet angle $\phi$ in the far-field. The FW-H predictions are then performed for all 36 microphones, and the resulting spectra are ensemble-averaged. Similar procedure is applied for the calculation of the Overall Sound Pressure Level (OASPL) in $\mathrm{dB}$, where the frequency range considered for the integration is $0.05 \leq S t \leq 3$. Finally, bin-averaged PSD is computed, with bin size $\Delta S t=0.05$, for both experimental and numerical data. To evaluate uncertainty on the experimental noise data, basic analysis was performed to estimate the errors due to the microphone sensitivity, the statistical errors during post-processing and the errors associated with measurements repeatability. The latter was found to be the main source of uncertainty, in general less than $0.5 \mathrm{~dB}$. As an indication of the error bounds, the minimum and maximum results obtained during the experimental campaign are shown by the error bars on the OASPL plot in figure 8 .

For the baseline cases $10 M$ and $64 M$, the noise spectra are reasonably well captured for most angles, up to frequency $S t \approx 1$. For higher frequencies however, the noise levels from these cases are systematically over-predicted. This is observed for both grids (i.e., independently of the refinement in the jet plume) and most inlet angles, in particular in the low angles $90^{\circ} \leq \phi \leq 120^{\circ}$, where the large-scale mixing noise is less dominant and the contribution of the other weaker noise sources becomes visible. For higher inlet angles like $\phi=150^{\circ}$, this high-frequency over-prediction is less severe but the peak radiation around $S t=0.2$ is slightly under-predicted. These trends translate into some discrepancies of approximately 1.5 to $2 \mathrm{~dB}$ in the OASPL predictions in figure 8 for both the low inlet angles and peak angles. 


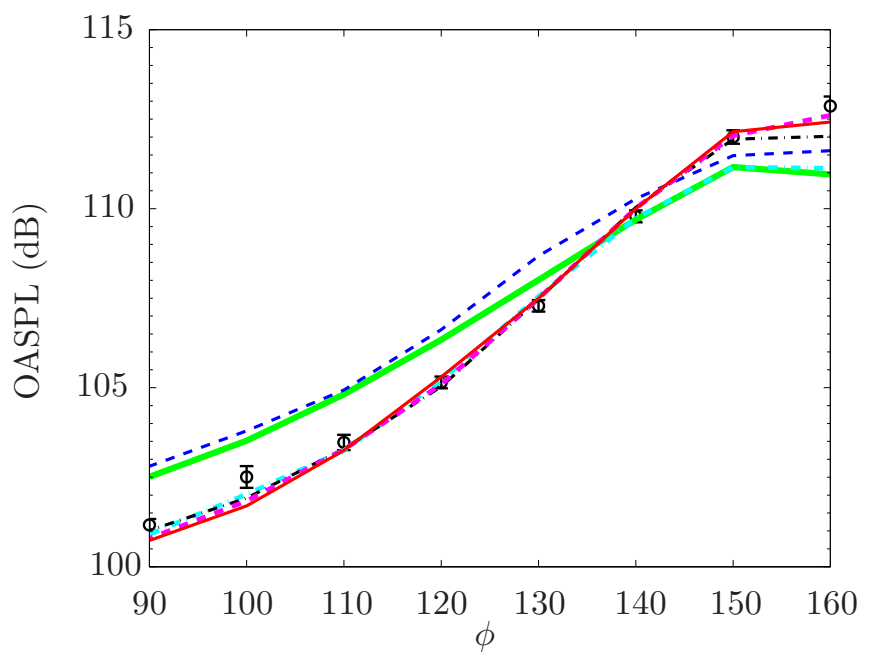

Figure 8. Overall sound pressure levels at $50 D$ from the nozzle exit from experiment ( $\circ$ ), baseline LES cases $10 M(----)$ and $64 M(\overline{(})$, and LES cases with modeling BL16M ( - - $)$, BL16M_Turb ( --- ), BL16M_WM $($ - - ) and BL16M_WM_Turb ( - ) .

Like the flow field results discussed in the previous section, it was found that the grid adaptation has the most significant impact on far-field noise predictions. With modeling, the over-prediction observed at high frequency for the baseline cases is eliminated. Excellent agreement with experimental measurements is obtained for all angles and frequencies up to $S t \approx 3$ on the present mesh. The resulting OASPL directivity curve in Figure 8 now lies within experimental uncertainty, with less than a $0.5 \mathrm{~dB}$ difference for most angles. The effects of synthetic turbulence and modeling on the predicted far-field noise are more subtle and would require more analysis. Arguably, the "best" match with experiment is however obtained for the case BL16M_WM_Turb with isotropic refinement of the boundary layer mesh inside the nozzle, wall modeling and synthetic turbulence (red curves in figures 7 and 8).

\section{Experimental and numerical databases}

Based on the preliminary LES study, the case BL16M_WM_Turb with isotropic refinement of the boundary layer mesh inside the nozzle, wall modeling and synthetic turbulence was down-selected to generate the long LES database. The total simulation time $t_{\text {sim }} c_{\infty} / D$ was extended to 2000 and the full LES flow field in primitive variable $(\rho, P, u, v, w)$ was collected every $\Delta t c_{\infty} / D=0.2$. Subsequently, additional simulation for the same configuration and numerical setup have been performed on the refined mesh (i.e., 69M cells), including the collection of the LES databases (see Table 1). In parallel, time-resolved PIV, 2-component LDV and acoustic measurements on the azimuthal array were conducted at PPRIME Institute. While postprocessing and analysis on the experimental and numerical databases are still currently ongoing, preliminary results and comparisons are presented in the next sections.

\section{A. Flow field}

Figure 9 shows the comparisons of the streamwise velocity statistics in the jet plume between PIV and LES for the two different grid resolutions. The corresponding centerline profiles are presented in figures 10. Despite the fact that the entire flow field was measured in five different acquisition location and field of views, a good continuity of the PIV velocity profiles can be observed in these figures. It can however be noticed that the RMS profiles from PIV in figure $10(b)$ shows an unexpected discontinuity around $x / D=2.5$. This can also be seen on the RMS field in the top row of figure $9(b)$, in the second measured area: a ring of higher RMS values can be observed surrounding the jet flow where very low RMS values are expected. This is attributed to artifacts introduced by the use of a lens of lower quality (i.e., non-macro) during this specific acquisition. This lens may have introduced high order distortions which could not be accounted for. 

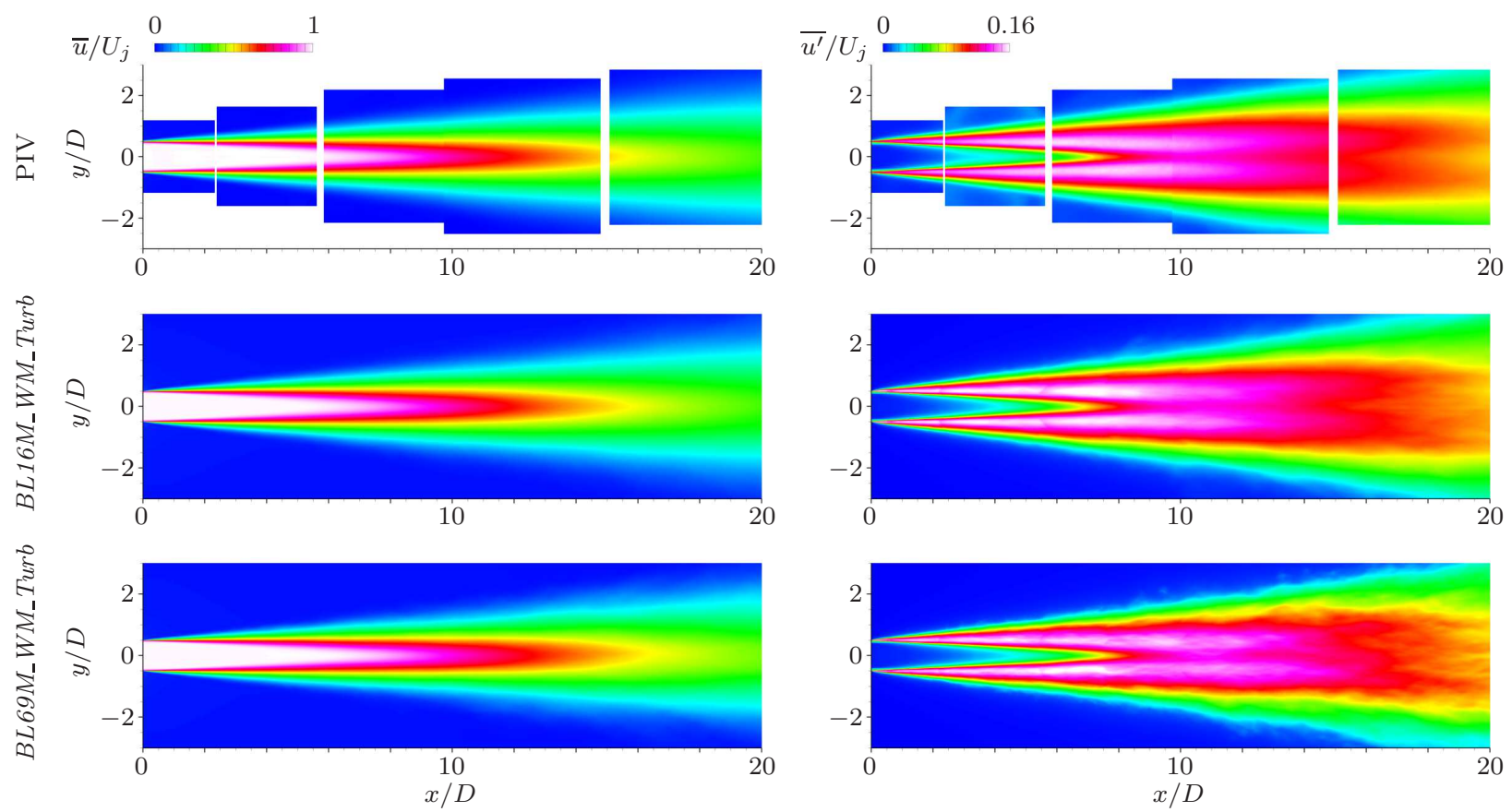

(a) Time-averaged streamwise velocity

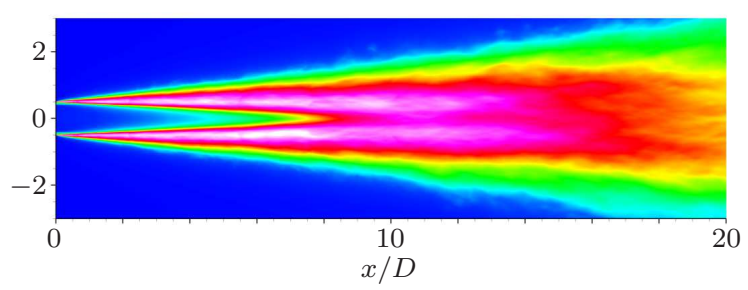

(b) RMS of streamwise velocity

Figure 9. Velocity statistics in the mid-section plane $(z=0)$ from experiment and from simulations with isotropic refinement, wall modeling and synthetic turbulence on the standard (16M) and refined (69M) grids.

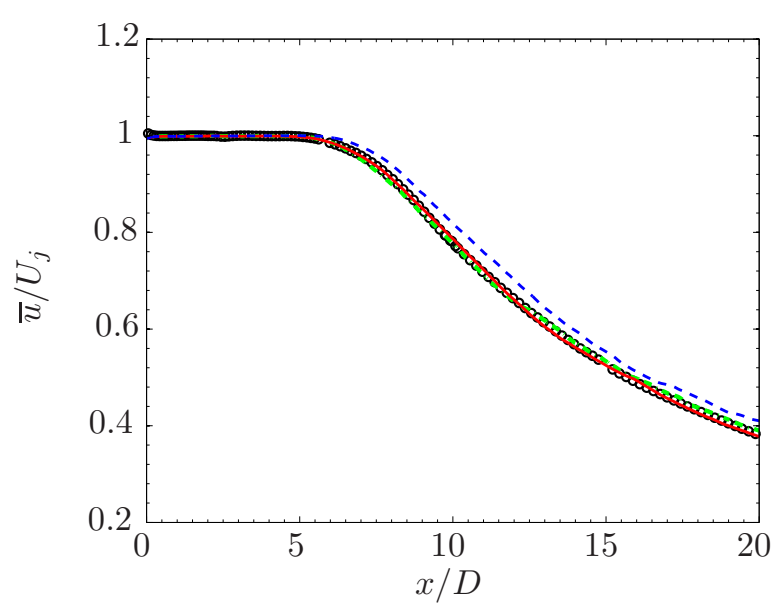

(a) Time-averaged streamwise velocity

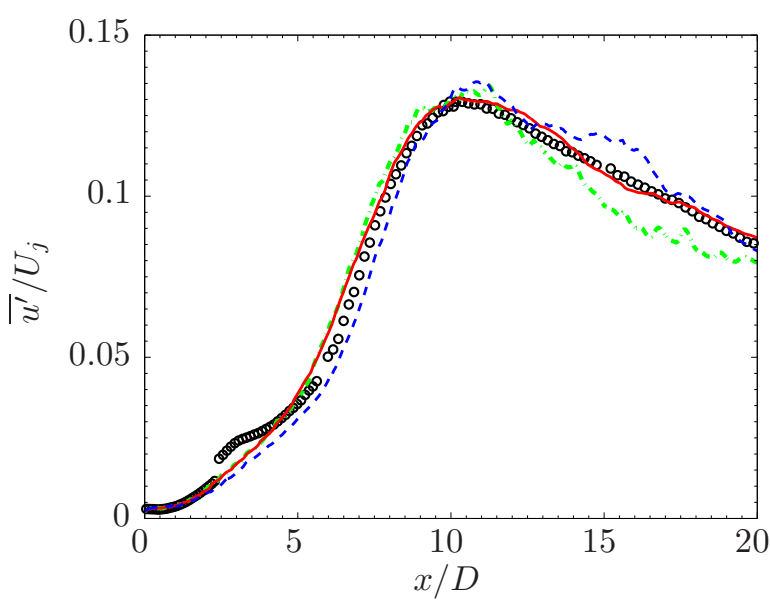

(b) RMS of streamwise velocity

Figure 10. Statistics of the streamwise velocity along the centerline from experiment ( $\circ$ ) and from LES cases with full modeling BL16M_WM_Turb $\left(-\right.$ for $t_{s i m} c_{\infty} / D=2000 ;--$ for $\left.t_{\text {sim }} c_{\infty} / D=300\right)$, and BL69M_WM_Turb (---- ).

Overall, there is a good agreement between the experimental and numerical results, for both mean and RMS streamwise velocity. The length of the potential core is well predicted by the LES, though, interestingly, a better match with experiment is obtained on the standard mesh. This quantity is challenging to capture, both numerically and experimentally, as it is sensitive to mesh resolution in the jet plume and to misalignment in the PIV measurements (i.e., based on the LES data, a rotation of $1^{\circ}$ from the jet axis starting at the nozzle exit would lead to a measured potential core length shorter by $0.7 D$ ).

Similarly, the velocity fluctuations along the centerline are well captured, in particular for the long LES database of case BL16M_WM_Turb. Due to the shorter simulation time, the refined case BL69M-WM_Turb shows more variations in the RMS levels in the downstream region of the jet plume $x / D>8$. Higherorder moments like RMS and skewness are typically more sensitive to temporal convergence and spatial resolution. The effect of short simulation time on these quantities is highlighted in figure $10(b)$ where the 
statistics computed over $t_{s i m} c_{\infty} / D=2000$ (i.e., , the total database duration) and 300 (i.e., , the duration from the preliminary study) are both reported for case BL16M_WM_Turb. While the mean centerline profiles are similar, significant variations are observed on the RMS profiles, with similar amplitude than the variations on the refined mesh. On the other hand, the mesh refinement in the jet plume for case BL69M_WM_Turb mitigates some the numerical artifacts associated with grid resolution. For instance, the slight discontinuities in RMS levels observed along the liplines at $x / D=2.5$ and 5 in figure $9(b)$ for case BL16M_WM_Turb correspond to unstructured grid transitions. While these transitions are still present for case BL69M_WM_Turb, the discontinuity in grid size is smaller and, as a result, the imprint on the RMS level is reduced.

\section{B. Acoustic field}

In addition to the single-microphone far-field noise, pressure measurements in the acoustic near-field were made using 18-microphone azimuthal ring arrays whose axial positions can be varied in order to map the sound field on a cylindrical surface of radius $r / D=14.3$ centered on the jet axis (see figure $1(b)$ ). Table 2 shows all the microphone coordinates and the complete comparison with the LES predictions is presented in figure 11. For the azimuthal array, both experimental and numerical PSD are averaged over the 18 microphones.

$\begin{array}{ccc}\mathbf{x} / \mathbf{D} & \mathbf{r} / \mathbf{D} & \phi \\ 0 & 14.3 & 90^{\circ} \\ 3.83 & 14.3 & 105^{\circ} \\ 8.25 & 14.3 & 120^{\circ} \\ 14.30 & 14.3 & 135^{\circ} \\ 17.04 & 14.3 & 140^{\circ} \\ 20.42 & 14.3 & 145^{\circ} \\ 24.77 & 14.3 & 150^{\circ} \\ 30.66 & 14.3 & 155^{\circ} \\ 39.29 & 14.3 & 160^{\circ}\end{array}$

$\begin{array}{ccc}\mathbf{x} / \mathbf{D} & \mathbf{r} / \mathbf{D} & \phi \\ 0 & 50.00 & 90^{\circ} \\ 8.68 & 49.24 & 100^{\circ} \\ 17.10 & 46.98 & 110^{\circ} \\ 25.00 & 43.30 & 120^{\circ} \\ 32.14 & 38.30 & 130^{\circ} \\ 38.30 & 32.14 & 140^{\circ} \\ 43.30 & 25.00 & 150^{\circ} \\ 46.98 & 17.10 & 160^{\circ}\end{array}$

(a) 18-microphone ring in the near-field

(b) Single microphone in the far-field

Table 2. Coordinates $x-r$ and corresponding inlet angle $\phi$ of the experimental microphones.

Overall, the agreement between experiment and simulation is excellent. For most inlet angles and relevant frequencies, the predictions are within $0.5 \mathrm{~dB}$ of the measurements, and consistent with the results from the preliminary LES study.

Aside from the slight variations in the very low frequencies due to temporal convergence, the main differences between the LES results from the two mesh resolutions are observed in the grid cut-off frequency for the high inlet angles $\phi \geq 150^{\circ}$ : at these angles, the limit frequency is about $S t \approx 2$ for the standard case BL16M_WM_Turb and $S t \approx 4$ for the refined case BL69M_WM_Turb with double the resolution in the jet plume. Preliminary analysis of the unstructured grid within the FW-H surface confirms that the cell spacing is approximately isotropic (as expected by design) and constant at $D / \Delta \approx 14$ in the standard mesh for $x / D>8$. Following the same procedure than in Refs. $25 \& 10$, and assuming an acoustic wave discretized with 8 points per wavelength, the limit frequency of acceptable resolution can be estimated at $S t_{\text {lim }}=D /\left(8 \Delta M_{a}\right) \approx 1.94$ for noise sources in the region $x / D>8$. This intermediate region after the end of the potential core, where the mixing layer flow transitions towards a fully-developed self-similar jet flow, is often regarded as the main region of sound generation for the dominant mixing noise. Changes in resolution in this region would therefore affect primarily the noise predictions at the peak angles $\phi=150-160^{\circ}$, consistent with the present observations.

For lower inlet angles, the high-frequency noise sources are typically expected between the nozzle and the end of the potential core, where the grid spacing is higher (namely $D / \Delta \approx 40-50$, and as high as 200 near the nozzle exit for the standard mesh), resulting in a higher limit frequency. As such, there are essentially no differences between the LES results at these angles. 


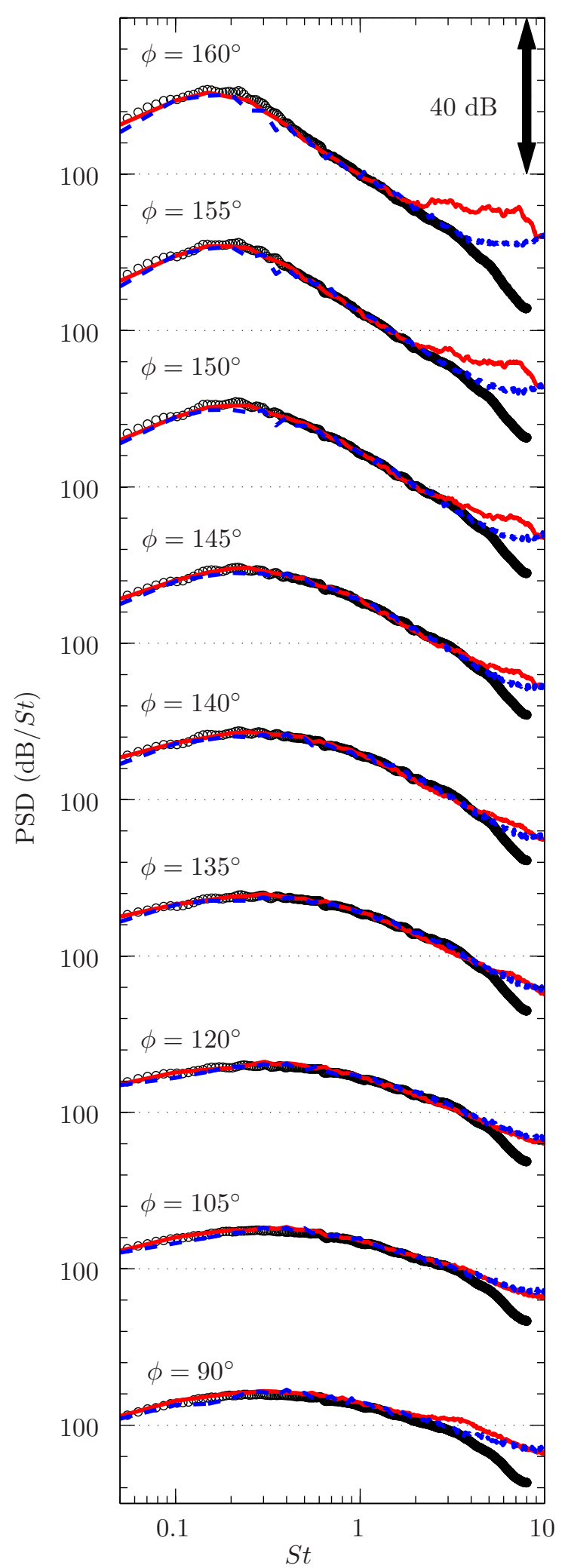

(a) Cylindrical array of radius $r=14.3 D$

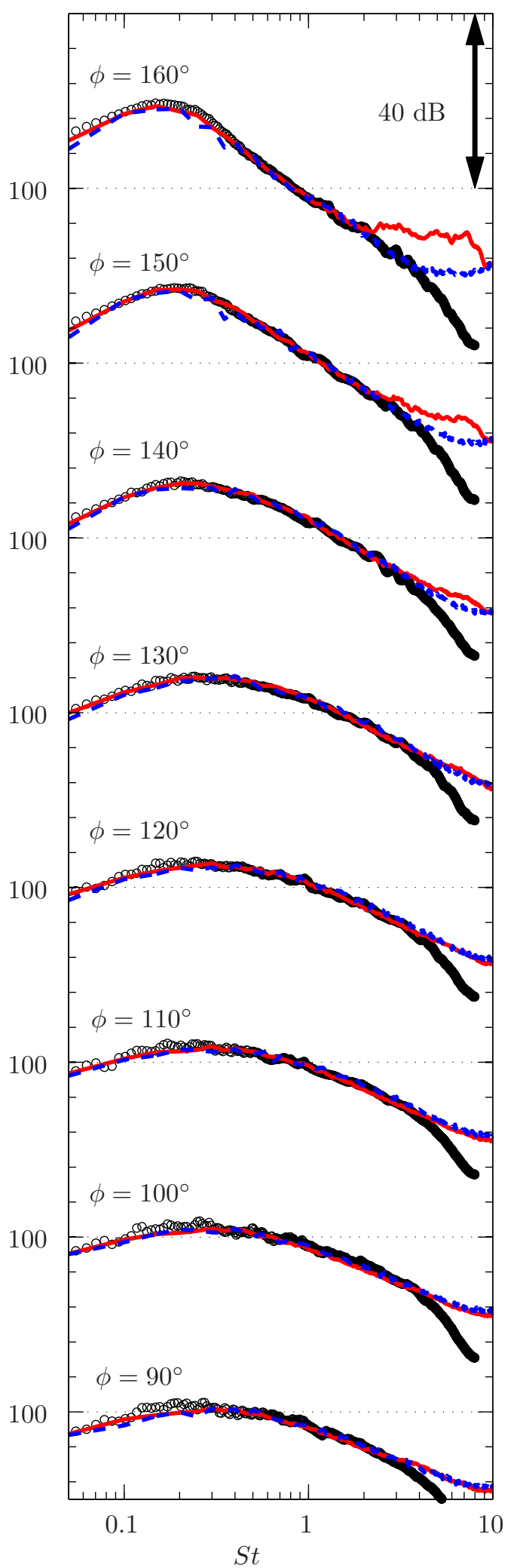

(b) Far-field array at constant distance $50 D$

Figure 11. Comparison of the noise spectra from experiment ( $\circ)$ and LES cases with full modeling BL16M_WM_Turb ( — ) and BL69M-WM_Turb (---- ) for all the experimental microphones. 


\section{Conclusions}

The focus of the present project was the generation of a high-fidelity jet database for noise modeling and wavepacket analysis. Large eddy simulations of a isothermal Mach 0.9 jet issued from contoured convergentstraight nozzle were performed, using the LES framework developed at Cascade Technologies. For all the cases considered, the nozzle geometry was explicitly included in the computational domain using unstructured body-fitted grids and the simulations were carried out at the experimental operating condition, including the full diameter-based Reynolds number $R e \approx 1 \times 10^{6}$.

Particular care was taken to ensure a fully turbulent jet and improve the nozzle interior unsteady flow modeling, seamlessly coupled with high-fidelity predictions of the jet plume and radiated noise. In preliminary work, a systematic parametric study of the effects of different modeling within the nozzle interior was conducted, focusing on localized adaptive mesh refinement inside the nozzle, application of synthetic turbulence, and wall modeling. Overall, the results show significant improvement for both flow field and noise predictions when modeling inside the nozzle was applied, compared to the typical approach based on coarse resolution in nozzle and laminar flow assumption commonly used in most jet simulations. With modeling, the nozzle-exit velocity statistics now exhibit fully turbulent profiles similar to the experimental data, and the far-field noise spectra now more closely match the measurements for all angles and most relevant frequencies. Note that the refinement inside the nozzle leads to only a small increase in grid size (i.e, about $6 \mathrm{M}$ cells), independent of resolution in jet plume and no change in the simulation time step. This results in a very modest increase of computational cost when all the different modelings are applied.

In terms of ranking in importance, the localized near-wall grid refinement inside the nozzle had the most significant impact on the flow field and radiated noise, followed by wall modeling. The addition of synthetic turbulence to model the boundary trip present in the experiment inside the nozzle also slightly improved the predictions, but overall the flow and noise showed little sensitivity to the details of the turbulence forcing for the present case. The same trend has been reported in the jet noise literature before and would tend to indicate the method can be made largely independent of tunable input parameters for the jet applications.

While previous attempts were made to simulate initially turbulent jets, ${ }^{26,27}$ this is the first time, to our knowledge, that a Mach 0.9 jet with fully turbulent nozzle-exit boundary layer has been successfully simulated, including flow field and far-field noise predictions. The resulting LES databases are currently being postprocessed and mined extensively to understand and model jet-noise source mechanisms. ${ }^{28,29}$

\section{Acknowledgments}

The preliminary LES studies were supported by NAVAIR SBIR project, under the supervision of Dr. John T. Spyropoulos. The main calculations were carried out on CRAY XE6 machines at DoD supercomputer facilities in ERDC DSRC. P. Jordan acknowledges the support of the French National Research Agency (ANR) through the project COOLJAZZ. Part of this work was performed during the Center for Turbulence Research Summer Program 2014 at Stanford University.

\section{Appendix}

Details of the noise post-processing procedure and non-dimensionalization are presented in the following sections. The experimental data is acquired at a frequency of $f_{a c}=200 \mathrm{kHz}$ (which corresponds to a Strouhal number, $\left.S t_{a c}=f_{a c} D / U_{j}=32\right)$. The acquisition runs for 20 seconds, and so each signal has $N=4 \times 10^{6}$ points.

The mean value is first removed:

$$
p(t)=P(t)-\frac{1}{N} \sum_{t=1}^{N} P(t) .
$$

Prior to down sampling the data is low-pass filtered via convolution with a three-point Gaussian, $w=$ $[0.25 ; 0.5 ; 0.25]$ :

$$
p_{L P}(t)=w(1) p(t-1)+w(2) p(t)+w(3) p(t+1) .
$$

This time-domain low-pass filter ensures that the down-sampled data is not aliased; the data is down-sampled 
at $f_{u}=100 \mathrm{kHz}\left(S t_{u}=16\right)$ and spectra are computed using this data.

Fast Fourier Transforms (FFT) are performed on blocks of data of size $N_{f f t}=2048$, and an overlap of $75 \%$ is imposed; i.e. block $i$, is

$$
N b_{i}=p_{L P}\left(1+(i-1) N_{o v}\right): p_{L P}\left(N_{f f t}+(i-1) N_{o v}\right)
$$

where

$$
N_{o v}=\frac{N_{f f t}}{4}-1
$$

A Hanning window is applied to each block prior to application of the FFT. A Fourier-transformed block is thus:

$$
\hat{p}(f)=\frac{\sqrt{8 / 3}}{N_{f f t}}\left(F F T\left(H(t) . * p_{L P}(t)\right)\right),
$$

where the factor $\sqrt{8 / 3}$ corrects for the energy loss associated with the Hanning window. The power spectral density of block $i$ is then computed as:

$$
\hat{P}_{i}(f)=\frac{2}{\Delta f} \hat{p}_{i}\left(f_{1}: \Delta f: f_{N y q}\right) \hat{p}_{i}^{*}\left(f_{1}: \Delta f: f_{N y q}\right),
$$

where $\Delta f=49 H z(\Delta S t \approx 0.008), f_{N y q}=50 k H z(S t \approx 8)$ and $\hat{p}_{i}^{*}(f)$ is the complex conjugate of $\hat{p}_{i}(f)$. The averaged narrowband power spectral density (PSD) is then

$$
\hat{P}(f)=\frac{1}{N b} \sum_{i=1}^{N b} \hat{P}_{i}(f),
$$

where $N b=3903$ is the total number of blocks of data.

In order for comparisons between simulations and experiments, or between experiments and experiments for that matter, to be valid, it is necessary to use appropriate non-dimensionalisation. The absolute jet conditions in the wind tunnel can vary from day to day, as the target control parameters are the dynamic Mach number, $M_{j}=U_{j} / c_{j}=0.9$ (as opposed to the acoustic Mach number, $M_{a}=U_{j} / c_{\infty}$ ) and a unitary temperature ratio, $T_{j} / T_{\infty}=1$. The ambient temperature in the anechoïc chamber, not being controlled, varies, as do, consequently, the jet velocity and temperature.

The following non-dimensionalisation of the measured data is thus performed. The power spectral density $\hat{P}(f)$ is first scaled so as to account for changes in the dynamic head of the jet:

$$
\frac{\hat{P}(f)}{\rho_{j}^{2} U_{j}^{4}}
$$

This quantity has units of $s^{-1}$, and its frequency dependence can be made non-dimensional by means of the factor $\frac{U_{j}}{D}$, (the power spectral density is now a function of the Strouhal number, $S t=\frac{f D}{U_{j}}$ ):

$$
\frac{\hat{P}(f)}{\rho_{j}^{2} U_{j}^{4}} \times \frac{U_{j}}{D}
$$

As this quantity is non-dimensional, calculation of the PSD in $d B / S t$ must be performed by means of a non-dimensional reference acoustic pressure level:

$$
P_{r e f}=\frac{P_{r e f}^{*}}{\rho_{\infty} c_{\infty}^{2}}
$$

where $P_{r e f}^{*}=20 \mu P a$ is the dimensional reference pressure. We thus have

$$
P S D=10 \log _{10}\left[\frac{\frac{\hat{P}(f)}{\rho_{j}^{2} U_{j}^{4}} \times \frac{U}{D}}{P_{\text {ref }}^{2}}\right]=10 \log _{10}\left[\frac{\hat{P}(f)}{P_{\text {ref }}^{*}{ }^{2}} \times \frac{U_{j}}{D} \times \frac{\rho_{\infty}^{2} c_{\infty}^{4}}{\rho_{j}^{2} U_{j}^{4}}\right]
$$

In the case of an isothermal jet, $\rho_{j} / \rho_{\infty}=1$, and $M_{a}=M_{j}$, such that the expression reduces to:

$$
P S D=10 \log _{10}\left[\frac{\hat{P}(f)}{P_{r e f}^{* 2}} \frac{U_{j}}{D} \frac{1}{M_{a}^{4}}\right]
$$




\section{References}

${ }^{1}$ Brès, G. A., Jordan, P., Colonius, T., Le Rallic, M., Jaunet, V., and Lele, S. K., "Large eddy simulation of a turbulent Mash 0.9 jet," Proceedings of the Summer Program, Center for Turbulence Research, Stanford University, 2014.

${ }^{2}$ Bradshaw, P., Ferriss, D. H., and Johnson, R. F., "Turbulence in the noise-producing region of a circular jet," J. Fluid Mech., 1964, pp. 591-624.

${ }^{3}$ Hill, G., Jenkins, R. C., and Gilbert, B. L., "Effects of the initial boundary-layer state on turbulent jet mixing," AIAA J., Vol. 14, No. 11, 1976, pp. 1513-1514.

${ }^{4}$ Bridges, J. E. and Hussain, A. K. M. F., "Roles of initial conditions and vortex pairing in jet noise," J. Sound Vib., Vol. 117(2), 1987, pp. 289-331.

${ }^{5}$ Bogey, C. and Bailly, C., "Influence of nozzle-exit boundary-layer conditions on the flow and acoustic fields of initially laminar jets," J. Fluid Mech., Vol. 663, 2010, pp. 507-538.

${ }^{6}$ Bogey, C., Mardsen, O., and Bailly, C., "Influence of initial turbulence level on the flow and sound fields of a subsonic jet at a diameter-based Reynolds number of $10^{5}, "$ J. Fluid Mech., Vol. 701, 2012, pp. 352-385.

${ }^{7}$ Brès, G. A., Ham, F. E., Nichols, J. W., and Lele, S. K., "Nozzle Wall Modeling in Unstructured Large Eddy Simulations for Hot Supersonic Jet Predictions," AIAA paper 2013-2142, 2013.

${ }^{8}$ Brès, G. A., Bose, S. T., Ham, F. E., and Lele, S. K., "Unstructured Large Eddy Simulations for Nozzle Interior Flow Modeling and Jet Noise Predictions," AIAA paper 2014-2601, 2014.

${ }^{9}$ Scarano, F., "Iterative image deformation methods in PIV," Meas. Sci. Tech., Vol. 13, 2002.

${ }^{10}$ Brès, G. A., Nichols, J. W., Lele, S. K., and Ham, F. E., "Towards Best Practices for Jet Noise Predictions with Unstructured Large Eddy Simulations," AIAA paper 2012-2965, 2012.

${ }^{11}$ Lockard, D. P., "An efficient, two-dimensional implementation of the Ffowcs Williams and Hawkings equation," J. Sound Vib., Vol. 229, 2000, pp. 897-911.

${ }^{12}$ Ffowcs Williams, J. E. and Hawkings, D. L., "Sound generation by turbulence and surfaces in arbitrary motion," Philosophical Transactions of the Royal Society of London. Series A, Mathematical and Physical Sciences, Vol. 264, 1969, pp. 321342.

${ }^{13}$ Brès, G. A., Nichols, J. W., Lele, S. K., Ham, F. E., Schlinker, R. H., Reba, R. A., and Simonich, J., "Unstructured Large Eddy Simulation of a Hot Supersonic Over-Expanded Jet with Chevrons," AIAA paper 2012-2213, 2012.

${ }^{14}$ Nichols, J. W., Lele, S. K., Moin, P., Ham, F. E., and Bridges, J. E., "Large-eddy simulation for supersonic rectangular jet noise prediction: effects of chevrons," AIAA paper 2012-2212, 2012.

${ }^{15}$ Nichols, J. W., Lele, S. K., and Spyropoulos, J. T., "The source of crackle noise in heated supersonic jets," AIAA paper 2013-2197, 2013.

${ }^{16}$ Freund, J. B., "Proposed Inflow/Outflow Boundary Condition for Direct Computation of Aerodynamic Sound," AIAA J., Vol. 35, No. 4, 1997, pp. 740-742.

${ }^{17}$ Mani, A., "Analysis and optimization of numerical sponge layers as a nonreflective boundary treatment," Journal of Computational Physics, Vol. 231, 2012, pp. 704-7016.

${ }^{18}$ Vreman, A., "An eddy-viscosity subgrid-scale model for turbulent shear flow: Algebraic theory and applications," Physics of Fluids, Vol. 16, Jan 2004, pp. 3570.

${ }^{19}$ Klein, M., Sadiki, A., and Janicka, J., "A digital filter based generation of inflow data for spatially developing direct numerical or large eddy simulations," Journal of computational Physics, Vol. 186, No. 2, 2003, pp. 652-665.

${ }^{20}$ Kawai, S. and Larsson, J., "Wall-modeling in large eddy simulation: Length scales, grid resolution, and accuracy," Phys. Fluids, Vol. 24(015105), 2012.

${ }^{21}$ Bodart, J. and Larsson, J., "Wall-modeled large eddy simulation in complex geometries with application to high-lift devices," Annual Research Briefs, Center for Turbulence Research, Stanford University, Stanford, CA, 2011.

${ }^{22}$ Bodart, J. and Larsson, J., "Wall-modeled large eddy simulation of the McDonnell-Douglas 30P/30N high-lift airfoil in near-stall conditions," AIAA paper 2012-3022, 2012.

${ }^{23}$ Shur, M. L., Spalart, P. R., and Strelets, M. K., "Noise prediction for increasingly complex jets. Part I: Methods and tests," International Journal of Aeroacoustics, Vol. 4, No. 3-4, 2005, pp. 213-246.

${ }^{24}$ Bogey, C., Mardsen, O., and Bailly, C., "Large-eddy simulation of the flow and acoustic fields of a Reynolds number $10^{5}$ subsonic jet with tripped exit boundary layers," Phys. Fluids, Vol. 23(035104), 2011.

${ }^{25}$ Mendez, S., Shoeybi, M., Sharma, A., Ham, F. E., Lele, S. K., and Moin, P., "Large-Eddy Simulations of PerfectlyExpanded Supersonic Jets Using an Unstructured Solver," AIAA J., Vol. 50, No. 5, 2012, pp. 1103-1118.

${ }^{26}$ Uzun, A. and Hussaini, Y. M., "Investigation of high frequency noise generation in the near-nozzle region of a jet using large eddy simulation," Theor. Comput. Fluid Dyn., Vol. 21(4), 2007, pp. 291-321.

${ }^{27}$ Bogey, C., Barré, S., and Bailly, C., "Direct computation of the noise generated by subsonic jets originating from a straight pipe nozzle," International Journal of Aeroacoustics, Vol. 7, No. 1, 2008, pp. 1-21.

${ }^{28}$ Jordan, P., Colonius, T., Brès, G. A., Zhang, M., Towne, A., and Lele, S. K., "Modeling intermittent wave packets and their radiated sound in a turbulent jet," Proceedings of the Summer Program, Center for Turbulence Research, Stanford University, 2014.

${ }^{29}$ Towne, A., Colonius, T., Jordan, P., Cavalieri, A. V. G., and Brès, G. A., "Stochastic and nonlinear forcing of wavepackets in a Mach 0.9 jet," AIAA paper 2015-2217, 2015. 